\title{
TAGUNG
}

\section{Selbstmarginalisierung eines Handels- und Werteimperiums}

\author{
Frédéric Krumbein*
}

Das Jahreskolloquium 2011 des Arbeitskreis Europäische Integration e.V. beschäftigte sich mit der Marginalisierung der Europäischen Union in der internationalen Politik. Die USA sind noch immer die einzige Weltmacht und unterhalten als einziger Staat ein globales Imperium. Die Europäische Union verfügt im Gegensatz dazu vor allem über wirtschaftliche Stärke und eine große Anziehungskraft ihrer Werte, die sie aber nur ungenügend in politischen Einfluss in der Welt umsetzen kann. ${ }^{1}$

Peter-Christian Müller-Graff begann mit einer Definition von Marginalisierung. Marginalisierung komme vom lateinischen ,margo“, der Rand, und meine die soziale Ausgrenzung bestimmter Gruppen. Beispiele für eine mögliche Marginalisierung Europas bestünden in der relativen Abnahme der europäischen Rüstungsausgaben im Vergleich zu denen Chinas, der Vereinigten Staaten oder Russlands.

\section{Der Blick der Schwellenländer auf Europa}

Natalia Chaban präsentierte die Ergebnisse eines Forschungsprojekts über die Wahrnehmung Europas in anderen Staaten der Erde. ${ }^{2}$ Sie stellte die Ergebnisse zur Wahrnehmung von Europa in China, Indien und Russland vor. Insgesamt seien die Wahrnehmungen in zwanzig Staaten untersucht worden. Das Projekt verfüge über drei Säulen. Die erste Säule bestehe aus einer Medienanalyse mehrerer Zei-

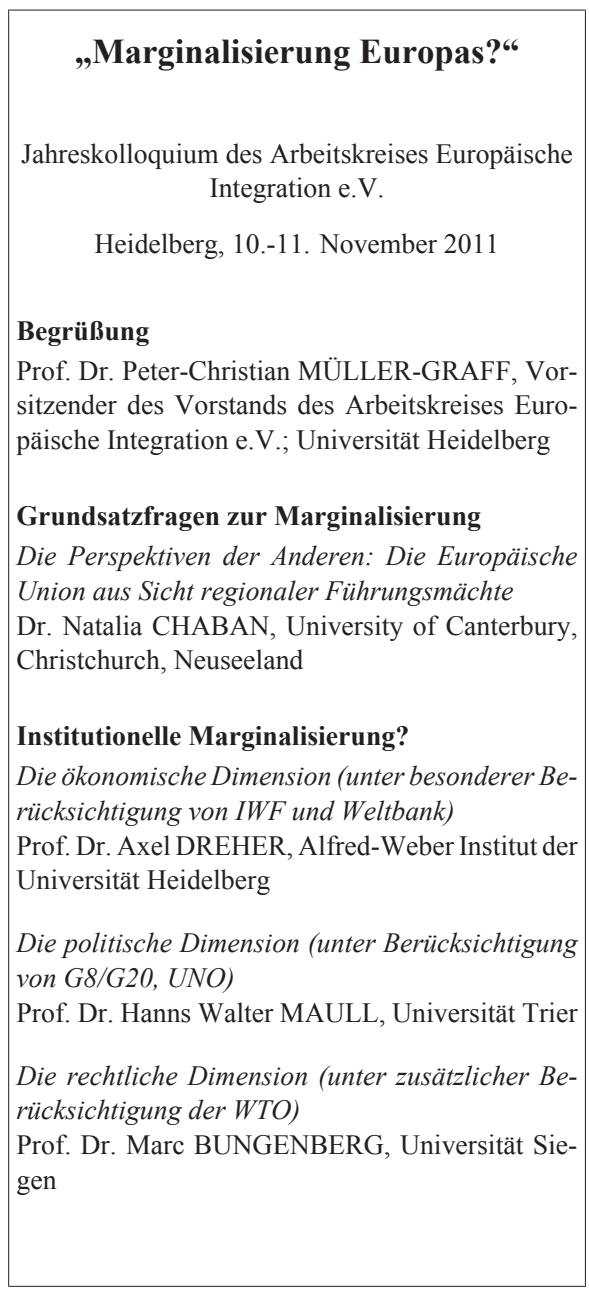

* Frédéric Krumbein, Geschäftsführer des Arbeitskreises Europäische Integration e.V., Berlin.

1 Der hier verwendete Begriff eines Imperiums ist angelehnt an Herfried Münkler, der als Charakteristika moderner Imperien unter anderem die Kontrolle über Kapitalströme, eine Leitwährung, technologische Innovationen und die Anziehungskraft für Migranten ansieht. Die Bedeutung von Werten als Machtfaktor in den internationalen Beziehungen wird von Joseph Nye unterstrichen. Vgl. Herfried Münkler: Imperien, Berlin 2005; Joseph Nye: Soft power, New York 2004.

2 „Perceptions of the EU in the Asia Pacific Region“. 
tungen und von Fernsehnachrichten. Die zweite Säule seien Meinungsumfragen und die dritte Säule stellten Interviews mit Entscheidungsträgern aus den Staaten dar. Ziel der Forschung sei es, das Verständnis der europäischen Identität in den verschiedenen Staaten zu bestimmen und zu evaluieren, ob Selbst- und Fremdbild übereinstimmten. So könne die Politik Europas angepasst werden, um Unterschiede im Selbst- und Fremdbild zu überbrücken.

Viele der befragten Wissenschaftler vermuteten, dass Europa nicht in der Lage sein werde, seine wirtschaftliche Stärke in politische oder militärische Macht umzusetzen. Europa sehe sich selbst oftmals als ethisches oder moralisches Projekt, zusammen gehalten durch Normen und die Attraktivität seiner Werte.

Chaban präsentierte weiterhin erste Ergebnisse der Medienanalyse. Im Vergleich zur Europäischen Union seien die USA in China, Indien und Russland als Akteur deutlich präsenter in den Medien und der Öffentlichkeit. In China werde von den drei Staaten am meisten über die Europäische Union berichtet. In der russischen und indischen Presse werde die Europäische Union hingegen selten erwähnt. Die Darstellung der Europäischen Union in chinesischen Publikationen sei die positivste von allen drei untersuchten Staaten. Die meisten Berichte seien aber wertfrei und bemühten sich um eine bloße Wiedergabe der Fakten.

In Asien insgesamt werde von den Angehörigen der europäischen Institutionen am häufigsten über den damaligen Präsidenten der Europäischen Zentralbank, Jean-Claude Trichet, berichtet, in China über die Hohe Vertreterin der Union für Außen- und Sicherheitspolitik Catherine Ashton (Hohe Vertreterin). Bundeskanzlerin Angela Merkel sei die am häufigsten genannte Politikerin in Asien und China. Deutschland stelle den am häufigsten erwähnten Staat in der Presse Asiens und Chinas dar.

\author{
Marginalisierung im Wirtschafts- und \\ Sicherheitsbereich? \\ Potentialwachstumsperspektive im globalen Ver- \\ gleich \\ Dr. Marcus KAPPLER, Zentrum für Europäische \\ Wirtschaftsforschung, Mannheim \\ Marginalisierung und Defizienzen in Sicherheit \\ und Verteidigung \\ Prof. Dr. Mathias JOPP, Direktor des Instituts für \\ Europäische Politik, Berlin \\ Europas neue Strategien der Selbstbehauptung \\ Tauglichkeit des neuen Lissabonner Rechtsrah- \\ mens für Welthandelsstrategie, Weltordnungspoli- \\ tik und Gemeinsame Außen- und Sicherheitspoli- \\ tik \\ Prof. Dr. Christoph VEDDER, Universität Augs- \\ burg \\ Europas Strategien im Umgang mit Schuldenkrisen \\ Dr. Andreas REES, Chefvolkswirt UniCredit, \\ München \\ Dreieckskooperationen als neue Strategie der \\ Selbstbehauptung der EU \\ Prof. Dr. Michèle KNODT und Nadine PIEFER, \\ Technische Universität Darmstadt
}

In der Analyse der Bilder in den Medien zu Europa zeigten sich ebenfalls interessante Ergebnisse. So hätte die indische Presse viele Bilder über Proteste in Griechenland publiziert. In China seien selten Protestbilder veröffentlicht worden. Hier stünden vor allem Bilder über fröhliche Politiker aus Europa und China im Vordergrund. Dies hänge offenbar mit dem autoritären Charakter des chinesischen politischen Systems zusammen. Die chinesische Presse berichte insgesamt eher positiv über Europa.

In der öffentlichen Meinung in den asiatischen Staaten wechselten sich China und die USA im Ranking der wichtigsten Staaten auf der Welt ab. Die Europäische Union werde meist auf den Plätzen fünf und folgende genannt. Allerdings erwarteten die Bevölkerungen in den meisten Staaten eine Zunahme der politischen Macht der Europäischen Union. Typische Begriffe, die mit der Europäischen Union assozi- 
iert würden, seien der Euro, Handel oder die Integration von Staaten.

Unter den Eliten der drei Staaten werde die Europäische Union in erster Linie als wirtschaftliche Macht wahrgenommen. Die Europäische Gemeinschaft werde als positives Beispiel zur Stärkung der eigenen Macht durch Kooperation betrachtet. In Indien spiele die normative Macht ebenfalls eine Rolle. In China werde die normative Macht der Europäischen Union nicht diskutiert. In China werde die zukünftige Welt als ein Machtdreieck aus China, den USA und Europa dargestellt. Indien sehe sich selbst als künftige wirtschaftliche Macht und als Vorbild für Europa. Europa könne von Indien lernen, weil Indien multikulturell und multireligiös sei und die Inder gut zusammenlebten. Russland betrachte sich selbst als eigenen Spieler, ohne dass die Notwendigkeit, Allianzen mit anderen Staaten zu schließen, bestehe.

Insgesamt sei das Wissen über die Europäische Union in Staaten außerhalb der Union gering. Die Mehrheit der öffentlichen Meinung und der Eliten glaube, dass die Europäische Union keine wichtige Rolle in Zukunft spielen werde.

\section{Der Einfluss der EU-Mitgliedstaaten in den internationalen Wirtschaftsinstitutionen}

Axel Dreher referierte über den Zusammenhang der Mitgliedschaft von Staaten im Sicherheitsrat der Vereinten Nationen und die Kreditvergabe an solche Staaten. Die USA, aber auch andere Staaten, wie Frankreich, vergeben laut Dreher mehr Entwicklungshilfe an Staaten, die im Sicherheitsrat der Vereinten Nationen nichtständige Mitglieder seien. Die Hilfe würde sich auf die zwei Jahre der Mitgliedschaft des Staates im Sicherheitsrat konzentrieren. Teilweise stiegen die Beiträge bereits ein bis drei Jahre vor der Mitgliedschaft, weil dann oftmals die kommende Zusammensetzung des Sicherheitsrates feststehe. Nach dem Ausscheiden aus dem Sicherheitsrat gebe es einen signifikanten Rückgang der finanziellen Leistungen. Dabei stehe bei der erwünsch- ten Zustimmung der temporären Mitglieder im Sicherheitsrat für die ständigen Mitglieder das Ziel einer Stärkung der Legitimität der von ihnen initiierten Resolutionen im Vordergrund. So wünschten sich die Vereinigten Staaten bei wichtigen Entscheidungen, wie einem geplanten Krieg, eine Rechtfertigung durch die Vereinten Nationen.

Die Staaten, die mit den USA stimmen, erhielten auch am meisten Kredite des Internationalen Währungsfonds (IWF). An Staaten, die gegen die USA stimmten, würden weniger Kredite des IWF vergeben. Nur wenn die USA und Europa sich uneins über die Vergabe von Krediten an bestimmte Staaten seien, dann erhalte laut Dreher die Verwaltung des IWF einen größeren Spielraum bei der Kreditvergabe. Wenn sich die USA und Europa einig seien, dann verfügten sie über ausreichende Stimmrechte und damit Macht im IWF, um über die Vergabe von Krediten eigenständig zu entscheiden.

Von einer Marginalisierung Europas im IWF und in der Weltbank könne keine Rede sein. Es gebe hier nur geringfügige Anpassungen der Stimmrechte, wie eine Zunahme des Stimmgewichts Chinas. Aber die beherrschende Koalition der USA und Europas in beiden Institutionen sei bislang unverändert und es gebe auch keine alternativen Koalitionen, zum Beispiel zwischen China und Indien. Trotzdem spielten ökonomische Kriterien, wie die Bedürftigkeit eines Staates eine wichtige Rolle bei der Kreditvergabe. Die politischen Faktoren, wie die Kooperation mit der Europäischen Union oder den USA, seien aber meist ausschlaggebend, wenn zwischen zwei Staaten zu entscheiden sei, wer Kredite bekommen solle.

\section{Europäische Union: uneinig und deshalb unwichtig}

Hanns Walter Maull sprach über die globale Macht Europas. Die Europäische Union sei die letzte Chance Europas auf globale Einflussnahme. Die Europäische Union könne als ein Typus ,sui generis“, ein neues postmodernes Modell im globalen System, betrachtet wer- 
den. Die Europäische Union schaffe es bislang nicht, ihrem wirtschaftlichen und politischen Gewicht in der Welt gerecht zu werden. Eines der positiven Beispiele europäischer Außenpolitik stelle die Befriedung des Balkans seit 1995 dar. Die EU-Politik gegenüber dem iranischen Nuklearprogramm könne als ein weiteres Beispiel angesehen werden. Dabei hänge der Einfluss aber immer von einer engen Koordination der Politik der Europäischen Union mit den USA ab.

Zwei Faktoren bestimmten Europas globalen politischen Einfluss: der Aufstieg anderer Staaten und die Strategie der Europäischen Union in ihrer Außen- und Sicherheitspolitik. Andere Staaten gewönnen an Macht und Gewicht im internationalen System. Weiterhin fehle Europa eine eigene kohärente und überzeugende Strategie in der Außen- und Sicherheitspolitik. Das Problem sei nicht die mangelnde Repräsentanz Europas in internationalen Institutionen. Europa verfüge über zahlreiche Vertreter in internationalen Gremien. Das Hauptproblem stelle die Diversität der Stimmen und die Uneinigkeit der europäischen Staaten auf der internationalen Ebene dar. Insgesamt sei Europa auf der globalen Ebene marginalisiert und verfüge über einen geringen Einfluss im internationalen System. Die unzureichende Reaktion auf den Umbruch in der arabischen Welt im Jahr 2011 sei ein Beispiel für den nicht existenten Einfluss. Eine Möglichkeit, um einheitlichere Positionen zu erzeugen, wäre einen gemeinsamen europäischen Sitz im Sicherheitsrat zu schaffen anstelle des englischen und des französischen ständigen Sitzes.

Ein effektiver Multilateralismus stelle Mittel und Ziel der Politik der Europäischen Union dar. Effektiv bedeute dabei, dass die Europäische Union es schaffe, das Verhalten anderer Akteure zu beeinflussen. Das Ideal sei eine verrechtlichte und auf der Charta der Vereinten Nationen basierende globale Ordnung. Eine multilaterale Politik stoße aber oftmals an ihre Grenzen. Ein Beispiel für eine gescheiterte multilaterale Politik der Europäischen Union sei die globale Klimapolitik. Das Problem der mangelnden Fähigkeit, selbst mit einer Stimme zu sprechen und sich zu einigen, mindere den Vorbildcharakter von multilateraler Politik als Ziel der Gemeinsamen Außen- und Sicherheitspolitik (GASP). Die Europäische Union wende normative Macht an, weil ihr die realpolitische Macht fehle. Die Förderung von Menschenrechten und Demokratie sei eine Konsequenz dieses normativen Machtanspruchs.

Die Europäische Union verkörpere ein technokratisches Modell der Zusammenarbeit von Eliten, welches zunehmend an seine Grenzen stoße. Das technokratische Modell bezeichne die Herrschaft wohlwollender Eliten zum Wohle der Prinzipale (Völker). Das Modell der Regierung Europas sei demokratisch nicht ausreichend legitimiert, sodass die Legitimität über die Resultate der europäischen Integration erfolge (,permissive consensus"). Die Gewinne an Produktivität und Wohlstand durch die letzten Erweiterungsrunden der Union seien aber gering gewesen, sodass die Schaffung von Legitimität für die Einheit Europas über die Resultate der europäischen Integration nicht mehr funktioniere. Die Europäische Union präsentiere sich aufgrund dieser inneren Schwächen insgesamt als ein wenig attraktives Modell von Multilateralismus.

Die Selbstmarginalisierung Europas könne nach Ansicht von Maull nur durch das Bemühen um innereuropäische Einigkeit und durch die Bildung von Allianzen mit anderen Mächten überwunden werden. Dann könnte gemeinsam Einfluss ausgeübt werden. Die EU-Politik im Konflikt um das iranische Nuklearprogramm verkörpere einen solchen Ansatz, der zwar schlussendlich nicht erfolgreich gewesen sei, aber in dem Europa sich einig zeigte, mit den USA zusammenarbeitete und es schaffte sogar Chinas Position - wenn auch nur geringfügig - zu verändern.

\section{Vielzahl der europäischen Stimmen in der Welthandelsorganisation}

Die Rolle der Europäischen Union im internationalen Wirtschaftsrecht war Gegenstand des 
Vortrags von Marc Bungenberg. Die DohaRunde der Welthandelsorganisation (WTO), in der bislang keine Einigung über neue Handelserleichterungen bestehe, und die nur bescheidenen Reformen im Internationalen Währungsfonds seien Beispiele für weitgehend gescheiterte Prozesse von "global governance“. Die Europäische Union trage Verantwortung für die Schaffung eines europäisch geprägten Weltordnungsrahmens. Diese Verantwortung finde sich in Art. 21 des Vertrags über die Europäische Union. In der internationalen Handelspolitik seien früher typische Streitfälle vor allem im transatlantischen Verhältnis aufgetreten. Inzwischen fänden sich Konflikte stärker im Verhältnis Europas zu anderen Staaten, wie im Verhältnis zu Brasilien, China, Indien oder Russland. Nach wie vor bestehe das große Problem für die externe Handlungsfähigkeit der Europäischen Union in der Unklarheit, welche EU-Institution auf der internationalen Ebene für Europa spreche. Gleichzeitig fallen wechselweise verschiedene EU-Mitgliedstaaten den anderen in den Rücken und verhinderten gemeinsame Positionen in der internationalen Politik.

Die Europäische Union besitze prinzipiell genügend Ressourcen, personell wie finanziell, um Einfluss in der globalen Handelspolitik auszuüben. Das wahre Gewicht stelle sich aber geringer dar. Die Europäische Union repräsentiere mehr als ein Sechstel der 153 Mitgliedstaaten in der WTO und verfüge damit über viele Stimmen, obwohl sie über einen einheitlichen Wirtschaftsraum verfüge. Es bestehe eine Doppelmitgliedschaft von Europäischer Union und den einzelnen EU-Mitgliedstaaten. Die anderen Mitgliedstaaten der WTO akzeptierten diese Doppelmitgliedschaft nur, weil in der WTO Entscheidungen grundsätzlich im Konsens gefunden werden.

\section{Die Wachstumsaussichten Europas}

Marcus Kappler sprach über die Beeinträchtigung des Produktionspotenzials im vierten Jahr der Finanz- und Schuldenkrise. Der mittel- bis langfristige Wirtschaftsausblick hänge stark von den künftigen potenziellen Produktionsmöglichkeiten ab. Das Produktionspotenzial stehe ebenfalls in einem engen Zusammenhang mit der Geldmenge. Wenn beispielsweise die Zinsen zu niedrig angesetzt würden und nicht dem Produktionspotenzial entsprächen, dann könne dies zu einer Erhöhung der Geldmenge, die nicht dem tatsächlichen Wachstum der Realwirtschaft entspreche, und dadurch zu Inflation führen.

Bei einer Krise gebe es drei mögliche Szenarien. Bei einem Wachstumseinbruch könne: Erstens, der alte Wachstumspfad wiedergefunden werden, das heißt das alte Niveau des Wirtschaftswachstums erreicht und der krisenbedingte Einbruch kompensiert werden; zweitens, die gleiche Wachstumsrate erreicht werden, aber auf dem niedrigeren Niveau der Krise; und drittens, ein niedrigeres Wachstum entstehen. Bankenkrisen in Schweden und Mexiko Anfang der 1990er Jahre seien Beispiele für das erste Szenario einer raschen Rückkehr zum alten Wachstumspfad. Japan und die Finanzkrise Anfang der 1990er Jahre sei ein Beispiel für das dritte Szenario, bei dem es zu einem dauerhaft niedrigeren Wachstum gekommen sei.

Ein Vergleich von Bankenkrisen in 152 Staaten zeige, dass erst sechs Jahre nach der jeweiligen Krise die Wirtschaft wieder auf einem Vorkrisenniveau wachse. Bei den Industriestaaten erhole sich das Wachstum nach etwa fünf Jahren. Es komme aber zu einem langfristigen Produktionsverlust. Das potenzielle Beschäftigungsniveau leide ebenfalls über einen längeren Zeitraum. Im Vergleich zu ,normalen' Rezessionen gebe es einen deutlich stärkeren Effekt. Vor allem das Beschäftigungsniveau sei bei einer ,normalen' Rezession sehr viel weniger betroffen als bei einer Bankenkrise. Finanzkrisen hätten negative und persistente Auswirkungen auf Kapital, Beschäftigung und Wachstumspotenzial.

Wenn man die ersten drei Szenarien auf Europa anwende, dann zeige sich, dass die Finanzkrise bei den großen europäischen Staaten, 
Deutschland und Frankreich, bislang relativ geringe Auswirkungen habe (Prognosen bis zum Jahr 2015). Der langfristige Wachstumspfad habe sich kaum geändert. Bei den südeuropäischen Staaten, Italien, Spanien, Portugal und Griechenland, gebe es einen starken Effekt auf den mittelfristigen Wachstumspfad (2011 bis 2015). Alle Staaten würden stark von ihrem Wachstumspfad abweichen. Italien befinde sich seit dem Jahr 2000 bereits unterhalb seines Wachstumspfades. Polen sei gut positioniert und verfüge auch mittelfristig über eine relativ stabile Volkswirtschaft. Für Großbritannien sei ebenfalls mittelfristig von einer leichten Erholung auszugehen. In den USA sei der Ausblick noch positiver, es sei mittelfristig eine vollständige Erholung der Volkswirtschaft zu erwarten.

\section{Vergrößerung der Kluft im militärischen Bereich zu anderen Staaten}

Mathias Jopp referiert über Defizite im Bereich der Gemeinsamen Sicherheits- und Verteidigungspolitik am Beispiel des LibyenKrieges 2011. Die GASP der Europäischen Union habe sehr gut bei der Verhängung von Sanktionen gegen das Regime von Muammar al-Gaddafi funktioniert, wie etwa dem gemeinsamen Waffenembargo. Die Europäische Union und ihre Organe seien aber insgesamt in der Libyen-Krise marginalisiert gewesen. Die Hohe Vertreterin, Catherine Ashton, und der Europäische Diplomatische Dienst hätten praktisch keine Rolle gespielt. Nationale Profilierungsabsichten hätten triumphiert. Die Europäische Union bleibe eine Zivilmacht und die Ausübung der militärischen Macht stelle weiterhin eine Prärogative der Einzelstaaten dar.

Wenn die Europäische Union in ihren internationalen Beziehungen militärische Macht anwenden wolle, dann brauche sie eine gemeinsame Verteidigungsstruktur. Großbritannien sei bislang der einzige Staat gewesen, der sich gegen einen weiteren Ausbau der Kapazitäten der Europäischen Union im Bereich der mili- tärischen Kooperation aussprach. Hier wäre die Frage, ob die anderen 26 Mitgliedstaaten den Mut haben, im Rahmen der ständigen strukturierten Zusammenarbeit, ohne Großbritannien eine Sicherheitsarchitektur in der Europäischen Union aufzubauen. Ohne eine solche Zusammenarbeit bleibe die Marginalisierung Europas bestehen.

Die USA seien Europa im militärischen Bereich weit überlegen. Die technologische Kluft zwischen den USA und Europa werde dort sogar immer größer. Die USA neigten zudem seit Ende des Kalten Krieges zu unilateralem Handeln und der Verwendung der $\mathrm{NATO}^{3}$ als „toolbox“, das heißt sie verwendeten die NATO nur bei Bedarf.

Inzwischen befänden sich die Vereinigten Staaten zunehmend auf dem Rückzug aus ihrem globalen militärischen Engagement. Gründe bestünden in den Erfahrungen vergangener Kriege, wie im Irak und in Afghanistan, und in budgetären Zwängen im Verteidigungshaushalt. Zwei Alternativen könne man skizzieren: ein stärkeres multilaterales Engagement oder ein Rückzug auf den eigenen Staat. Vermutlich würden die USA einem dritten Szenario folgen und, wie der ehemalige Verteidigungsminister Robert Gates sagte, zwischen „wars of necessity" und „wars of choice“ unterscheiden. Wenn es notwendig sei, würden die USA weiterhin ihre militärische Macht zur Durchsetzung der eigenen Interessen nutzen. Ein stärkeres multilaterales Engagement mit den Europäern zusammen sei aber unwahrscheinlich.

\section{Bestehendes Potenzial zur Selbstbehauptung, aber mangelnder Wille}

Der Vortrag von Christoph Vedder beschäftigte sich mit den Kompetenzen und Verfahren der Europäischen Union und den Möglichkeiten nach dem Vertrag von Lissabon zur Selbstbehauptung der Union im internationalen System. Der Vertrag von Lissabon und seine

3 North Atlantic Treaty Organization. 
Strukturen taugten als Gerüst für eine Strategie der Selbstbehauptung der Europäischen Union im internationalen System. Inwieweit dieses Gerüst mit Leben gefüllt werde, hänge von der Politik ab.

Die GASP habe mit regelmäßigen Konsultationen der Außenminister begonnen. Bis in die 1980er Jahre habe es nur zwei außenpolitische Kompetenzen der Europäischen Gemeinschaft gegeben: die gemeinsame Handelspolitik und Assoziationsabkommen mit anderen Staaten. Die GASP umfasse inzwischen die gesamte Außenpolitik der Union mit Ausnahme der Beziehungen zu anderen Staaten, die zu den vollständig vergemeinschafteten Politikfeldern gehören, wie beispielsweise Handelsbeziehungen. In der GASP verkörpere die Hohe Vertreterin, Catherine Ashton, die primäre Vertretung der Europäischen Union nach außen. Nur auf der Ebene der Staats- und Regierungschefs repräsentiere der Präsident des Europäischen Rates die Union in ihren auswärtigen Angelegenheiten. Die Hohe Vertreterin vertrete die Europäische Union in allen anderen Bereichen mit Ausnahme der Handelspolitik.

\section{Die Schuldenkrise im Fokus Europas}

Andreas Rees sprach über Europas Umgang mit der Schuldenkrise und über eine wichtige Institution zur Unterstützung des Euro durch die European Financial Stability Facility (EFSF), die notleidenden Mitgliedstaaten der Eurozone Kredite zur Verfügung stellt. Ihr Volumen reiche nicht aus, um die beste Bewertung als Schuldner, ein AAA-Rating, zu erhalten. Dies liege daran, dass inzwischen zu wenige Staaten in der Eurozone selbst ein AAARating hätten und die EFSF aber von allen Staaten der Eurozone, die meisten mit einem schlechteren Rating, getragen werde. Durch eine Hebelwirkung sei das Volumen der EFSF auf 1.500 Milliarden Euro ausgedehnt worden. Italien benötige im Jahr 2012 etwa 300 Milliarden Euro an neuen Krediten und Spanien würde etwa 100 Milliarden Euro neu an Krediten aufnehmen müssen. Die EFSF sei ausreichend zur Absicherung dieser neuen Kredite und könne Italien und Spanien für zwei Jahre die Refinanzierung ihrer Schulden ermöglichen, sollten beide Staaten keinen Zugang zu den Kapitalmärkten erhalten.

Rees erläuterte, dass es rational schwer zu erklären sei, warum die Zinsen für italienische Anleihen in den letzten Monaten so stark angestiegen seien, da sich keine Veränderung in der wirtschaftlichen Situation vollzogen habe. Die Lohnstückkosten seien seit dem Jahr 2000 in einigen Staaten, wie Griechenland oder Irland, stark angestiegen und die Staaten hätten über ihre Verhältnisse gelebt. In Italien sei dies so nicht der Fall gewesen, die Lohnstückkosten seien kaum gestiegen. Die öffentliche Verschuldung in Italien sei sehr hoch, die private Verschuldung hingegen niedrig. Das Niveau der privaten Verschuldung sei vergleichbar mit jenem in Deutschland und Frankreich. Außerdem verfüge der öffentliche Haushalt über einen Primärüberschuss, das heißt einen Überschuss im Haushalt abzüglich der Zinszahlungen.

Zur Konsolidierung würden die betroffenen Staaten erhebliche Anstrengungen unternehmen. Portugal und Irland verfügten über Sparpläne und -anstrengungen sowie Steuererhöhungen in den Jahren 2011 bis 2013 mit einem Volumen von über 10 Prozent des Bruttoinlandsprodukts (BIP) der Staaten. Griechenland wolle seine Staatsausgaben um etwa 8 Prozent kürzen und Spanien um etwas über 6 Prozent. Frankreich plane ein Sparprogramm von etwas mehr als 2 Prozent des BIP. Dies stelle das ambitionierteste Sparprogramm der französischen Geschichte dar und solle trotz anstehender Präsidentschaftswahlen in 2012 umgesetzt werden.

In den USA verschiebe sich die fiskalische Konsolidierung auf die Jahre nach 2013, was zu einer Abstufung des Ratings der US-Staatsanleihen führen könnte. Die Langzeitarbeitslosigkeit in den USA sei seit Beginn der Finanzkrise stark angestiegen. Der US-Arbeitsmarkt stelle sich nicht mehr so flexibel wie in früheren Zeiten dar. 45 Millionen US-Ameri- 
kaner hätten inzwischen ein Anrecht auf Essensmarken. Im Jahr 2000 seien dies deutlich weniger als 20 Millionen gewesen.

\section{Die Zusammenarbeit Europas mit \\ Schwellenstaaten bei der Vergabe von Entwicklungshilfe}

Michèle Knodt und Nadine Piefer referierten über Kooperationen zwischen der Europäischen Union als Geber von Entwicklungshilfe, anderen neuen Geberstaaten und begünstigten Staaten. Diese Kooperationen zielten auf die Selbstbehauptung der Europäischen Union auf der internationalen Ebene. Schwellenstaaten würden vermehrt als Geber in Drittstaaten auftreten und verfolgten eigene strategische Ziele in ihrer Außenpolitik. Teilweise verfügten Schwellenstaaten über Wettbewerbsvorteile in Weltregionen, wie beispielsweise in Afrika.

Es gebe verschiedene Szenarien der Zusammenarbeit zwischen den drei Akteuren. Beispielsweise könne die Zusammenarbeit aus einer bestehenden Kooperation eines traditionellen Gebers mit einem begünstigten Staat entstehen. Motive der neuen Geber für Kooperationen seien die Übernahme internationaler Verantwortung, das gegenseitige Lernen oder die Nutzung der Entwicklungszusammenarbeit als neues außenpolitisches Instrument. Motive der traditionellen Geber bestünden unter anderem in dem Wunsch nach einem stärkeren Einfluss in Afrika, einer Erhöhung der Mittel der Entwicklungshilfe durch Kooperation, einer Harmonisierung der Politik der Geber und der Ausnutzung komplementärer Stärken der verschiedenen Geberstaaten.

Für Empfängerländer seien Vorteile die doppelte Expertise der Europäischen Union und der Schwellenstaaten, die Replizierbarkeit von Programmen in der eigenen Entwicklungszusammenarbeit, die bereits erfolgreich in den Schwellenstaaten umgesetzt wurden, eine höhere Effizienz bei der Nutzung von Mitteln der
Entwicklungshilfe oder der Zugriff auf europäisches Know-how. Das Dreieck sei schließlich eine stärkere Struktur als eine bilaterale Zusammenarbeit: Falls ein Partner wegfalle, blieben immer noch zwei Partner übrig und könnten die Kooperation fortsetzen. Bestehende Herausforderungen bei Dreieckskooperationen seien beispielsweise unterschiedliche normative Voraussetzungen, wie in der Kooperation der Europäischen Union mit China. Im Zeitraum 2013 bis 2018 seien im Rahmen der Entwicklungshilfe der Europäischen Union 50 bis 60 Millionen Euro als finanzielle Mittel für Dreieckskooperationen vorgesehen.

\section{Die Einheit als notwendiges Ziel Europas}

Insgesamt wurde bei dem Jahreskolloquium deutlich, dass Europa in der Weltpolitik unter seinen Möglichkeiten bleibt. Europa verfügt über die wirtschaftlichen und finanziellen Ressourcen, um einen stärkeren globalen Einfluss auszuüben. Weiterhin ist Europa in den globalen Institutionen, wie dem UN-Sicherheitsrat oder dem IWF, sehr gut repräsentiert.

Das Hauptproblem der Europäischen Union liegt in der fehlenden Einheit Europas. Zu oft kann sich Europa nicht einigen und vertritt deshalb entweder keine oder schwache Positionen auf der internationalen Ebene. Hinzu kommt eine Krise des europäischen Regierungssystems, dem es zunehmend an Akzeptanz und Legitimität unter den Bürgern mangelt, und die Schuldenkrise als akute Bedrohung der europäischen Integration und des Wohlstands Europas. Eine Vertiefung des europäischen Integrationsprozesses, mit der Etablierung einer echten Fiskal- und Wirtschaftsunion und einer Reform der politischen Institutionen, würde nicht nur die Schuldenkrise lösen, sondern bietet auch die Chance die Selbstmarginalisierung Europas auf der globalen Ebene und die Mängel des europäischen Regierungssystems zu beseitigen. 